\title{
Effect of injection energy on residual dose around the charge exchange foil
}

\author{
Kazami Yamamoto \\ Japan Atomic Energy Agency (JAEA), Tokai, Ibaraki, 319-1195, Japan \\ Shinichi Kato \\ Tohoku University, Sendai 980-8578, Japan \\ (Received 12 June 2012; published 10 December 2012)
}

\begin{abstract}
The rapid cycling synchrotron (RCS) of Japan Proton Accelerator Research Complex (J-PARC) and the accumulator ring (AR) of Spallation Neutron Source (SNS) can be used as high-power pulsed neutron sources. In both cases, the injection region becomes one of the highest activation areas in the ring. However, residual dose distributions have revealed that the highest activation points in the J-PARC RCS and the SNS AR are different in detail. The dose of the charge exchange chamber in the SNS is more than 100 times larger than that of the RCS though the ratio of beam power is less than 10 . We investigated the reason of this difference by GEANT4 and MARS, and the calculation results indicated that the difference was due to the dependence of the neutron and pion production rate on the injection energy.
\end{abstract}

DOI: 10.1103/PhysRevSTAB.15.120401

PACS numbers: 29.27.Ac

\section{INTRODUCTION}

In recent years, two proton accelerator systems have been used as high-power pulsed neutron sources with the time structure of the order of a few microseconds. One is a rapid cycling synchrotron (RCS) into which a beam at hundreds of millions of electronvolts is injected, and the beam is accelerated to several gigaelectronvolts. The other, an accumulator ring (AR), does not accelerate but makes a pulsed structure in microseconds. In the AR, a linac accelerates the beam up to the GeV energy. While Japan Proton Accelerator Research Complex (J-PARC) [1] and ISIS [2] adopt the RCS system, Spallation Neutron Source (SNS) [3] and Los Alamos Neutron Science Center (LANSCE) [4] use the AR system. In both cases, a charge exchange system, which includes the painting process of the phase space, is used for injection from the linac to the ring in order to mitigate the space charge effect [5]. So far, a charge exchange foil is needed to strip two electrons efficiently from an $\mathrm{H}^{-}$beam. As a result, the injection region becomes one of the highest activation areas in the ring because of the interaction between the injection and circulating beam and the charge exchange foil. However, residual dose distributions have revealed that the highest activation points in the J-PARC RCS and the SNS AR are different.

In the SNS AR, the vacuum chamber of the charge exchange foil is the highest activation point. After 1 month, 1 MW output, and 2 days of cooling, the residual dose value is about $9 \mathrm{mSv} / \mathrm{h}$ at the position of $30 \mathrm{~cm}$ from the

Published by the American Physical Society under the terms of the Creative Commons Attribution 3.0 License. Further distribution of this work must maintain attribution to the author(s) and the published article's title, journal citation, and DOI. chamber surface [6]. In the J-PARC RCS, the branch of the HO dump line and the beam position monitor installed downstream of the H0 dump branch are the highest activation points. The dose values on the chamber surfaces are about $1.5 \mathrm{mSv} / \mathrm{h}$ after 1 month, $120 \mathrm{~kW}$ output, and $5 \mathrm{~h}$ of cooling [7]. It became clear that the losses in the RCS were caused by the scattering of the injection and circulating beams at the foil. The residual dose at the charge exchange foil chamber of the RCS is about $140 \mu \mathrm{Sv} / \mathrm{h}$ on the chamber surface and tens of $\mu \mathrm{Sv} / \mathrm{h}$ at the $30 \mathrm{~cm}$ position under the same conditions. The dose of the charge exchange chamber in the SNS is about 100 times larger than that of the RCS though the ratio of beam power is less than 10.

In order to investigate the reason of this difference, we used GEANT4 [8] to calculate the secondary particles created in the foil. Afterward, we used the MARS code [9] to estimate the influence of those secondary particles on the dose of the charge exchange chamber. We used J-PARC parameters as the basis for these calculations.

\section{CAlCulation}

\section{A. GEANT4}

When the injection or circulating beam hits the foil, secondary particles are produced at the foil as a result of interactions between the beam and foil atoms. Because only GEANT4 can calculate thin material like this foil, the parameters of those secondary particles were calculated by the GEANT4 code.

In this calculation, since the energy of the two convoy electrons accompanying the injection proton was not very high (since relativistic $\beta$ of $1 \mathrm{GeV}$ proton is 0.88 , the two convoy electrons have the same $\beta$-then the electron energy is $550 \mathrm{keV}$ for the $1 \mathrm{GeV} \mathrm{H}^{-}$case), we neglected 


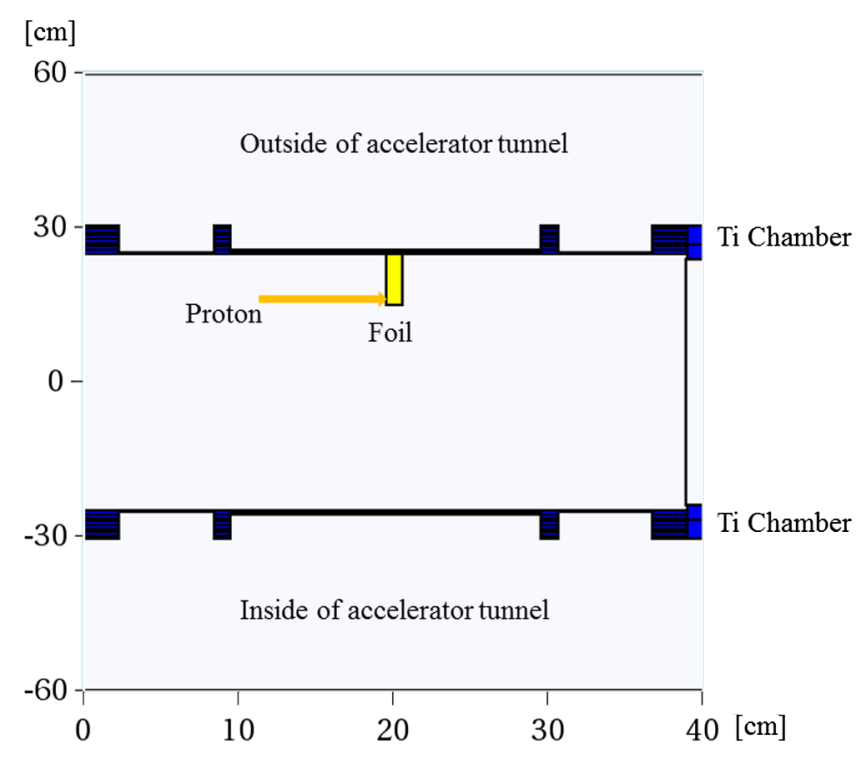

FIG. 1. Geometry of MARS calculation. In this figure, the charge exchange foil (yellow) is expanded to clarify its position.

those electrons and regarded the injection beam as only protons. The injection beam was assumed to be a pencil beam.

Since the differences in the foil collision area and the angle between the injection beam and the circulating beam are small, we assumed that the distributions of the circulating beam and the injection beam were the same.

We used $10^{9}$ protons as an input to GEANT4 and estimated the production rate of each secondary particle. We performed calculations at three injection energies:
(1) $181 \mathrm{MeV}$, the present condition of J-PARC; (2) $400 \mathrm{MeV}$, a planned upgrade for J-PARC; and $1 \mathrm{GeV}$, in imitation of the SNS case. The foil is made of carbon and its thickness is $200 \mu \mathrm{g} / \mathrm{cm}^{2}$ (in the $181 \mathrm{MeV}$ case), $300 \mu \mathrm{g} / \mathrm{cm}^{2}$ (in the $400 \mathrm{MeV}$ case), and $350 \mu \mathrm{g} / \mathrm{cm}^{2}$ (in imitation of the SNS case). From the conditions of actual operation and the simulation, the average number of one proton collision with the foil could be evaluated to be about 10 in J-PARC. Here we assumed the average number of one proton collision with the foil is ten in all cases.

\section{B. MARS}

Using the GEANT4 output parameters (trajectory and energy of secondary particles), we estimated with MARS the residual dose on the foil chamber. The calculation model in MARS is shown in Fig. 1. In this model, the foil chamber is assumed to be a cylinder of $400 \mathrm{~mm}$ length and $500 \mathrm{~mm}$ inner diameter. The material is titanium, and the foil is $150 \mathrm{~mm}$ away from the circulating beam center. In the MARS calculation, the numbers of secondary particle were calculated by multiplying the number of collided proton and the average number of generated secondary particle per one proton together. Here, the average number of generated secondary particle per one proton was the result from GEANT4. In all injection energy cases, the number of injected protons was assumed to $2.08 \times 10^{14}$ per second. This corresponds to the number of particles of $100 \mathrm{~kW}$ output by $3 \mathrm{GeV}$ extraction energy. Therefore the number of protons that collide with the foil was estimated to be $2.08 \times 10^{15}$ per second.

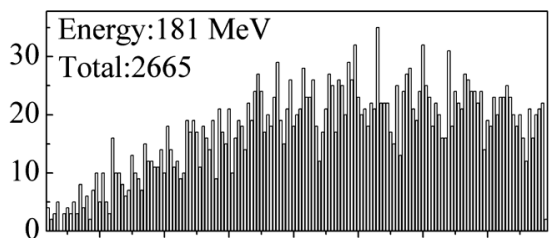

$\begin{array}{lllllll}0.2 & 0.4 & 0.6 & 0.8 & 1.0 & 1.2 & 1.4\end{array}$
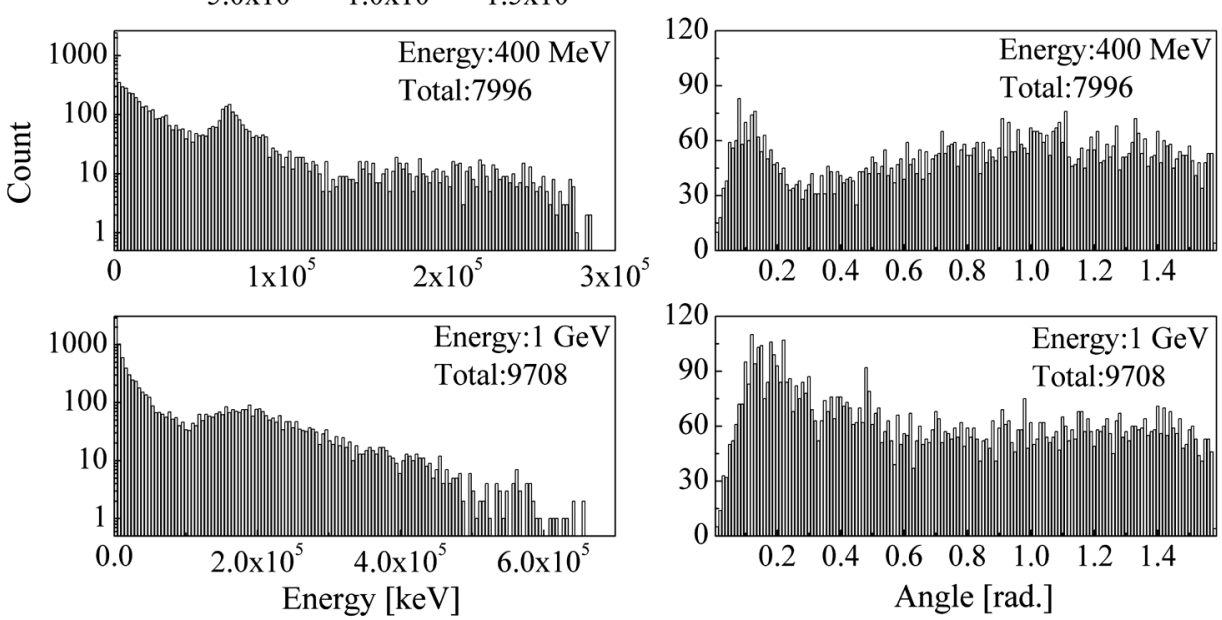

FIG. 2. Energy and angular spectra of the neutrons produced at the foil. 

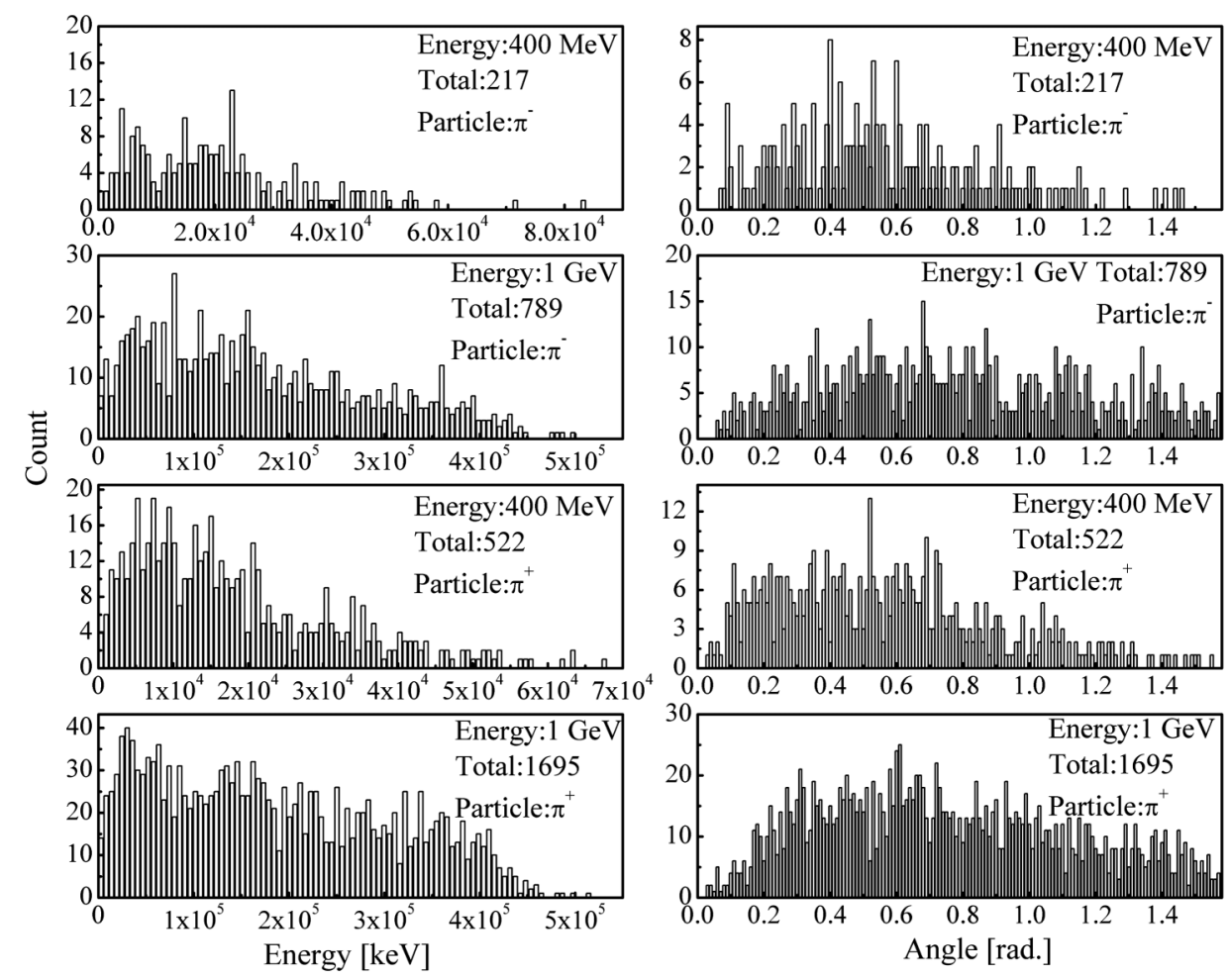

FIG. 3. Energy and angular spectra of pions produced at the foil.

\section{RESULTS AND DISCUSSION}

\section{A. GEANT4}

From the GEANT4 calculation, secondary gamma rays, electrons, pions, and neutrons were produced in these cases. Among these, the energies of the gamma rays and electrons were not sufficiently high to cause a nuclear reaction; therefore, we neglected these particles. The energy and angle spectra of the neutrons and pions are shown in Figs. 2 and 3. In these figures, the angle value was defined at the angle to the circulating beam center. The numbers of total production by $10^{9}$ protons are shown in each graph. Neutrons were generated in all cases, and the number of neutrons increased with the energy of the primaries. When the injection energy was higher than $400 \mathrm{MeV}$, the energy spectra had a peak at $70 \mathrm{MeV}$ for the $400 \mathrm{MeV}$ injection energy and at $190 \mathrm{MeV}$ for the $1 \mathrm{GeV}$ injection energy. Concerning the angular spectra, the neutrons in the forward direction increased with the energy of the primaries.
Since the threshold energy for pion production is $280 \mathrm{MeV}$, no pions were included in the $181 \mathrm{MeV}$ injection energy calculation. The $400 \mathrm{MeV}$ protons of the $10^{9}$ total were able to produce only hundreds of pions, and those energies were less than $100 \mathrm{MeV}$. But when the injection energy was $1 \mathrm{GeV}$, the number of total pions was more than 1000 and their energy reached $500 \mathrm{MeV}$.

The results, shown in Table I, indicate that the number of neutrons from a $400 \mathrm{MeV}$ injection was 3 times as large as that from a $181 \mathrm{MeV}$ injection, and the number of neutrons from a $1 \mathrm{GeV}$ injection was about 4 times larger. When the injection energy was $1 \mathrm{GeV}$, pions and antipions were also generated, with their total number being $23 \%$ of the number of neutrons.

By using the GEANT4 results, we made input files containing the particle energy, position, and momentum. The numbers of secondary particle were calculated by multiplying the number of collided proton and the average number of generated secondary particle per one collided proton together.

TABLE I. Production number of secondary particles.

\begin{tabular}{lcrr}
\hline \hline & The number of neutrons/proton & The number of $\pi^{-} /$proton & The number of $\pi^{+} /$proton \\
\hline $181 \mathrm{MeV}$ & $2.67 \times 10^{-6}$ & & \\
$400 \mathrm{MeV}$ & $8.00 \times 10^{-6}$ & $2.17 \times 10^{-7}$ & $5.22 \times 10^{-7}$ \\
$1 \mathrm{GeV}$ & $9.71 \times 10^{-6}$ & $7.89 \times 10^{-7}$ & $1.70 \times 10^{-6}$ \\
\hline \hline
\end{tabular}


TABLE II. Residual dose rate around the foil.

\begin{tabular}{lccc}
\hline \hline & $181 \mathrm{MeV}$ & $400 \mathrm{MeV}$ & $1 \mathrm{GeV}$ \\
\hline Dose by neutron (errors) $[\mathrm{mSv} / \mathrm{h}]$ & $7.92 \times 10^{-2}$ & $1.83 \times 10^{-1}$ & $1.96 \times 10^{-1}$ \\
& $\left(5.75 \times 10^{-3}\right)^{\mathrm{a}}$ & $\left(2.05 \times 10^{-3}\right)^{\mathrm{a}}$ & $\left(1.99 \times 10^{-3}\right)^{\mathrm{a}}$ \\
& $\left(2.43 \times 10^{-4}\right)^{\mathrm{b}}$ & $\left(5.37 \times 10^{-4}\right)^{\mathrm{b}}$ & $\left(6.67 \times 10^{-4}\right)^{\mathrm{b}}$ \\
Dose by $\pi^{-}[\mathrm{mSv} / \mathrm{h}]$ & & $1.87 \times 10^{-4}$ & $3.59 \times 10^{-2}$ \\
& & $\left(2.10 \times 10^{-5}\right)^{\mathrm{a}}$ & $\left(1.51 \times 10^{-3}\right)^{\mathrm{a}}$ \\
& & $\left(6.78 \times 10^{-5}\right)^{\mathrm{b}}$ & $\left(7.69 \times 10^{-5}\right)^{\mathrm{b}}$ \\
Dose by $\pi^{+}[\mathrm{mSv} / \mathrm{h}]$ & $4.81 \times 10^{-4}$ & $5.70 \times 10^{-2}$ \\
& & $\left(1.27 \times 10^{-5}\right)^{\mathrm{a}}$ & $\left(1.43 \times 10^{-3}\right)^{\mathrm{a}}$ \\
& & $\left(2.91 \times 10^{-5}\right)^{\mathrm{b}}$ & $\left(1.24 \times 10^{-4}\right)^{\mathrm{b}}$ \\
Total dose rate (error) $[\mathrm{mSv} / \mathrm{h}]$ & $7.92 \times 10^{-2}$ & $1.84 \times 10^{-1}$ & $2.89 \times 10^{-1}$ \\
& $\left(5.76 \times 10^{-3}\right)$ & $\left(2.12 \times 10^{-3}\right)$ & $\left(2.96 \times 10^{-3}\right)$ \\
\hline \hline
\end{tabular}

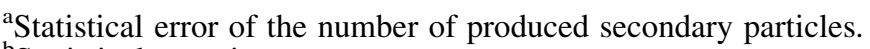

${ }^{\mathrm{b}}$ Statistical error in MARS.

\section{B. MARS}

The MARS code was used to estimate the residual dose on the surface of the foil chamber. Table II shows the calculation results of MARS. These values represent the residual dose after 30 days of operation with proton foil hits of $2.08 \times 10^{15}$ per second and 1 day of cooling.

In a $181 \mathrm{MeV}$ injection, pions were not produced, and only neutrons contributed to the dose. When the injection energy was increased to $400 \mathrm{MeV}$, some pions and antipions were generated. However, the contribution of pions was only $0.4 \%$ of the dose because their amount is less than neutrons and their energy is too small.

At an injection energy of $1 \mathrm{GeV}$, not only neutrons but also pions contributed to the residual dose. Because of the increment of the neutron production rate and the contribution of pions, the residual dose was about 3.6 times as large as the value from the $181 \mathrm{MeV}$ injection case. In this calculation, we assumed $2.08 \times 10^{15}$ foil hits per second which corresponds to $100 \mathrm{~kW}$ extracted beam power at $3 \mathrm{GeV}$ extracted beam energy. A $1 \mathrm{GeV}$ extraction energy would require 3 times more injected particles, and 3 times more foil hits, which would increase the residual activation by an additional factor of 3. By taking into account these effects, residual dose becomes approximately 1 order of magnitude larger. Although the geometry in the calculation was modeled for the J-PARC RCS, this result could explain the reason for the higher activation in SNS.

\section{CONCLUSION}

When the injection energy is less than $400 \mathrm{MeV}$, neutrons are the main source of the residual dose at the chamber near the foil. The neutron production rate becomes larger as the injection energy is increased, and this causes even more activation. If the injection energy is further increased and becomes nearly $1 \mathrm{GeV}$, not only the increment of the neutron production rate but also the additional production of pions bring about even more activation of the chamber.

These results indicate that maintainability and radiation hardness of the components near the foil should be taken into account in the design of the higher injection energy system. On the other hand, experience at the J-PARC RCS shows that low injection energy causes large angle scattering of protons in the foil. To reduce losses from these scattered particles, countermeasures should be considered in the design of the lower injection energy system. In the J-PARC RCS, an additional collimation system has been installed downstream of the foil $[10,11]$. Therefore, we should change a point to notice according to the injection energy.

[1] M. Kinsho, in Proceedings of the International Particle Accelerator Conference, Kyoto, Japan (ICR, Kyoto, 2010), MOPEC069.

[2] D. J.S. Findlay, in Proceedings of the 2007 Particle Accelerator Conference, Albuquerque, New Mexico (IEEE, New York, 2007), TUYKI01.

[3] J. Galambos, in Proceedings of HB2010 (2010), MOIB01.

[4] R. W. Garnett, M.S. Gulley, K.W. Jones, and J. L. Erickson, in Proceedings of LINAC2010 (2010), TUP025.

[5] H. Harada, KEK Report. No. 2009-7.

[6] J. Galambos, in Proceedings of HB2010 (2010), MOIB01.

[7] K. Yamamoto et al., in Proceedings of IPAC'10 (2010), WEPEB065.

[8] GEANT4 home page, http://geant4.cern.ch/index.shtml.

[9] MARS home page, http://www-ap.fnal.gov/MARS/.

[10] K. Yamamoto et al., in Proceedings of IPAC'11, TUPS033.

[11] S. Kato et al., in Proceedings of IPAC'12, MOPPD074. 